\title{
Colloidal plasmonic gold nanoparticles and gold nanorings: shape-dependent generation of singlet oxygen and their performance in enhanced photodynamic cancer therapy
}

This article was published in the following Dove Press journal:

International Journal of Nanomedicine

\author{
Yamin Yang' \\ Yue $\mathrm{Hu}^{2}$ \\ Henry Du ${ }^{3}$ \\ Lei Ren ${ }^{4}$ \\ Hongjun Wang ${ }^{5}$ \\ 'Department of Biomedical \\ Engineering, Nanjing University \\ of Aeronautics and Astronautics, \\ Nanjing, Jiangsu, China; ${ }^{2}$ Department \\ of Biological and Environmental \\ Engineering, Cornell University, Ithaca, \\ NY, USA; ${ }^{3}$ Department of Chemical \\ Engineering and Materials Sciences, \\ Stevens Institute of Technology, \\ Hoboken, NJ, USA; ${ }^{4}$ Department of \\ Biomaterials, College of Materials, \\ Xiamen University, Xiamen, Fujian, \\ China; ${ }^{5}$ Department of Biomedical \\ Engineering, Chemistry and Biological \\ Sciences, Stevens Institute of \\ Technology, Hoboken, NJ, USA
}

Correspondence: Yamin Yang Department of Biomedical Engineering, Nanjing University of Aeronautics and Astronautics, No 29 jiangjundadao,

Nanjing, Jiangsu, China 21 II 06

Tel +862584892368

Email yaminyang@nuaa.edu.cn

Hongjun Wang

Department of Biomedical Engineering,

Stevens Institute of Technology,

Castle Point on Hudson, Hoboken,

NJ 07030, USA

Tel +l 20I 2165556

Fax + I 20I 2168240

Email hongjun.wang@stevens.edu

\begin{abstract}
Introduction: In recognition of the potentials of gold nanoparticles (Au NPs) in enhanced photodynamic therapy (PDT) for cancer, it is desirable to further understand the shape-dependent surface plasmonic resonance (SPR) properties of various gold nanostructures and evaluate their performances in PDT.
\end{abstract}

Materials and methods: Monodispersed colloidal spherical solid Au NPs were synthesized by UV-assisted reduction using chloroauric acid and sodium citrate, and hollow gold nanorings (Au NRs) with similar outer diameter were synthesized based on sacrificial galvanic replacement method. The enhanced electromagnetic (EM) field distribution and their corresponding efficiency in enhancing singlet oxygen $\left({ }^{1} \mathrm{O}_{2}\right)$ generation of both gold nanostructures were investigated based on theoretical simulation and experimental measurements. Their shape-dependent SPR response and resulted cell destruction during cellular PDT in combination with 5-aminolevulinic acid (5-ALA) were further studied under different irradiation conditions.

Results: With comparable cellular uptake, more elevated formation of ${ }^{1} \mathrm{O}_{2}$ in 5-ALA-enabled PDT was detected with the presence of Au NRs than that with Au NPs under broadband light irradiation in both cell-free and intracellular conditions. As a result of the unique morphological attributes, exhibiting plasmonic effect of Au NRs was still achievable in the near infrared (NIR) region, which led to an enhanced therapeutic efficacy of PDT under NIR light irradiation.

Conclusion: Shape-dependent SPR response of colloidal Au NPs and Au NRs and their respective effects in promoting PDT efficiency were demonstrated in present study. Our innovative colloidal Au NRs with interior region accessible to surrounding photosensitizers would serve as efficient enhancers of PDT potentially for deep tumor treatment.

Keywords: gold nanoparticles, colloidal gold nanorings, surface plasmonic resonance, photodynamic therapy

\section{Introduction}

The unique optical properties and associated surface plasmonic resonance (SPR) effects of gold $(\mathrm{Au})$ nanostructures have proven useful for multifaceted biomedical applications including bioimaging, biomolecule sensing, thermally modulated drug delivery, photothermal cancer therapy, photodynamic therapy (PDT), etc. ${ }^{1-5}$ Typically, gold nanomaterials exhibit collective electron charge oscillations named localized surface plasmon resonances (LSPRs), through which the optical absorption and scattering can be dramatically enhanced with elevated local near-field amplitude at the resonance frequency. Such LSPR properties greatly depend on the particle shape, 
size, and dielectric environment. ${ }^{6-9}$ With recent advances that allow the controlled synthesis and dedicate fabrication of gold materials on a nanoscale, the plasmonic response of Au nanostructures can be tuned for different purposes. ${ }^{10}$

The cell destruction mechanism underlying the photodynamic reaction combines the activation of photosensitizer (PS) molecules under light irradiation, generation of cytotoxic reactive oxygen species (ROS) (mainly composed of singlet oxygen, ${ }^{1} \mathrm{O}_{2}$ ) following energy transfer from activated PS to surrounding oxygen molecules, and ROS-triggered cell death via the apoptosis and/or necrosis pathway. ${ }^{11}$ The active involvement of gold nanomaterials in PDT could offer at least two advantages over other nanomaterial-based drug delivery systems: significantly elevated ${ }^{1} \mathrm{O}_{2}$ generation and increased treatment depth for deep tumors. ${ }^{12,13}$ Our previous findings have highlighted that, in addition to delivering more PS to cells, plasmonic gold nanomaterials could significantly amplify the electromagnetic (EM) field and elevate ${ }^{1} \mathrm{O}_{2}$ formation, which consequently led to more destruction of cancer cells. ${ }^{14-18}$ Furthermore, by modulating the morphology and geometry, the plasmonic absorbance of Au nanostructures can be tuned into near-infrared (NIR) regions, allowing PDT for deep tumors under NIR light irradiation. ${ }^{19-21}$

In our previous studies, we have successfully synthesized various $\mathrm{Au}$ nanostructures with different sizes, ${ }^{22}$ opposite surface charges, different surface modifications, mitochondria-targeting capability, ${ }^{15}$ and tunable structure and controllable dimensions, ${ }^{17}$ and have tested their utility for enhanced PDT of cancer. In particular, we have theoretically and experimentally analyzed the size-dependent and surface charge-dependent enhancement of ROS formation enabled by spherical solid gold nanoparticles (Au NPs). We have also utilized our novel colloidal gold nanorings (Au NRs) as a drug delivery device and PS enhancer in PDT. ${ }^{23,24}$ In the present work, we further compared two types of gold nanostructures (Au NPs and Au NRs) with identical surface composition and outer diameter to investigate the shape-dependent SPR response during PDT. Particular emphasis was made to elucidate their corresponding efficiency in enhancing ${ }^{1} \mathrm{O}_{2}$ formation due to the elevated EM field under different irradiation conditions, including the use of NIR light as a light source.

\section{Materials and methods Materials}

Cobalt chloride hexahydrate $\left(\mathrm{CoCl}_{2} \cdot 6 \mathrm{H}_{2} \mathrm{O} 99.99 \%\right)$, sodium borohydride $\left(\mathrm{NaBH}_{4} 99 \%\right)$, sodium citrate trihydrate, gold(III) chloride solution ( $30 \mathrm{wt} \%$ of $\mathrm{HAuCl}_{4}$ in dilute $\mathrm{HCl}$ ), poly(vinylpyrrolidone) (PVP) (weight-average molecular weight=2,500 g/mol), 5-aminolevulinic acid (5-ALA), and protoporphyrin IX (PpIX) were purchased from SigmaAldrich Co. (St Louis, MO, USA). The deionized water used in this study was 18.2 M $\Omega$ Milli-Q filtered by Quantum Ex, Ultrapure Oranex Cartridge filtration columns (EMD Millipore, Billerica, MA, USA).

\section{Synthesis of Au nanostructures and characterization}

$\mathrm{Au}$ NRs were synthesized based on a sacrificial galvanic replacement method, in which cobalt nanoparticles were used as the template. The detailed synthesis process was described in our previous reports. ${ }^{14,18,23,24}$ Briefly, $100 \mu \mathrm{L}$ of $0.4 \mathrm{M}$ $\mathrm{CoCl}_{2} \cdot 6 \mathrm{H}_{2} \mathrm{O}$ and $400 \mu \mathrm{L}$ of $0.1 \mathrm{M}$ sodium citrate trihydrate were mixed with $100 \mathrm{~mL}$ Milli-Q water. The solution was deaerated with a continuous argon flow for $40 \mathrm{~min}$. Then, $1 \mathrm{~mL}$ of freshly prepared $0.1 \mathrm{M} \mathrm{NaBH}_{4}$ and $200 \mu \mathrm{L}$ of $1 \%$ (weight/volume [w/v]) PVP (molecular weight=2,500 g/mol) were simultaneously added to the solution under vigorous stirring to obtain cobalt nanoparticles. Subsequently, $150 \mu \mathrm{L}$ of $0.1 \mathrm{M} \mathrm{HAuCl}_{4}$ was dropwise added to the cobalt nanoparticle solution and kept stirring for another $30 \mathrm{~min}$. Then, the solution was exposed to the ambient environment, allowing for oxidization of unreacted cobalt. Solid Au NPs were synthesized using a modified Lee and Meisel method with the aid of ultraviolet (UV) irradiation. Briefly, 1\% (w/v) aqueous sodium citrate $(4 \mathrm{~mL})$ was added to $\mathrm{HAuCl}_{4}$ solution $(5 \mathrm{mM}$, $40 \mathrm{~mL}$ ). The mixture was then irradiated with UV for $30 \mathrm{~min}$ under stirring. To ensure both Au nanostructures bear the same surface chemistry, the obtained Au NPs were also coated with PVP by mixing $1 \mathrm{~mL}$ PVP solution $(1 \mathrm{mg} / \mathrm{mL})$ with $9 \mathrm{~mL}$ synthesized Au NPs under constant stirring overnight. To remove free PVP, the reacting solution was centrifuged under 10,000 rpm for $10 \mathrm{~min}$ and washed with Milli-Q water twice. A transmission electron microscope (TEM) (Philips CM20; FEI, Hillsboro, OR, USA) was used to examine the size and morphology of the prepared Au nanostructures. The plasmon resonances of prepared $\mathrm{Au}$ nanostructures were measured using a UV-visible multimode microplate reader (Synergy ${ }^{\mathrm{TM}}$ HT; BioTek Instruments, Inc., Winooski, VT, USA) with $1 \mathrm{~nm}$ resolution. The size and zeta-potential values of Au nanostructures were measured using laser Doppler electrophoresis with a Zetasizer (Nano-ZS; Malvern Instruments, Malvern, UK).

\section{Plasmonic simulation of $\mathrm{Au}$ nanostructures}

Computational simulation of plasmonic properties of Au NPs and Au NRs was performed using the discrete dipole approximation (DDA) algorithm implemented in the DDSCAT 
7.3 code developed by Draine and Flatau. ${ }^{25,26}$ Target Au nanostructures were respectively constructed based on the captured TEM images of Au NPs and Au NRs. BLENDER3D (version 2.78; Blender Foundation, www.blender.org) was used to generate a triangular mesh file of the 3D target geometries in this study. This file was then converted to a DDA compatible shape file using the online tool DDSCAT Convert (available at nanohub.org). The overall target is composed of 33,552 dipoles for Au NPs and 44,416 dipoles for $\mathrm{Au}$ NRs. The optical spectra and near-field distribution of $\mathrm{Au}$ nanostructures were calculated using the dielectric constant of bulk Au taken from Johnson and Christy. ${ }^{27}$

\section{Measurement of ' $\mathrm{O}_{2}$ generation}

The probe of Singlet Oxygen Sensor Green reagent (SOSG; Sigma-Aldrich Co.) could emit detectable green fluorescence in the presence of ${ }^{1} \mathrm{O}_{2}$, and thus SOSG was used to measure ${ }^{1} \mathrm{O}_{2}$ generation with $\mathrm{Au}$ NPs or $\mathrm{Au}$ NRs under different conditions. Briefly, $50 \mu \mathrm{L}$ of Au nanostructure solution with the same gold concentration $(2 \mathrm{mM})$ was mixed with $50 \mu \mathrm{L}$ of $10 \mu \mathrm{M}$ PpIX. Then, $50 \mu \mathrm{L}$ of $10 \mu \mathrm{M}$ SOSG was added to the mixture under dark conditions with a total volume of $150 \mu \mathrm{L}$. Samples in 96-well plates were then irradiated with broadband light, $600 \mathrm{~nm}$ long pass (LP) filtered light, and $700 \mathrm{~nm}$ LP filtered light for different time durations. The fluorescence intensity was measured $1 \mathrm{~min}$ after irradiation using a multimode microplate reader (Synergy HT; BioTek Instruments, Inc.) at an excitation wavelength of $485 / 20 \mathrm{~nm}$ and an emission wavelength of 528/20 nm.

\section{Cell culture and cytotoxicity test}

The triple-negative human breast adenocarcinoma cell line MDA-MB-231 (American Type Culture Collection [ATCC], Manassas, VA, USA) was used for cellular PDT tests. MDA-MB-231 cells have been reported for their resistance to traditional chemotherapies, aggressive pathological features, and high rates of metastasis and recurrence, and stand as a suitable in vitro model for evaluating PDT efficiency. ${ }^{28,29}$ MDA-MB-231 cells were cultured in Leibovitz L-15 Medium (Sigma-Aldrich Co.) supplemented with 10\% fetal bovine serum and $1 \%$ penicillin/streptomycin in a humidified incubator at $37^{\circ} \mathrm{C}$ without $\mathrm{CO}_{2}$. Media were refreshed every 2 days and the cells were passaged at a confluence of $70 \%-80 \%$. For cytotoxicity evaluation, cells were incubated with $\mathrm{Au}$ NRs or Au NPs at the same gold concentration $(40 \mu \mathrm{M}$, $80 \mu \mathrm{M}$, and $160 \mu \mathrm{M}$, respectively) for $24 \mathrm{~h}$, and the cell viability was determined using the MTT (Sigma-Aldrich Co.) assay. Briefly, cells after different treatments were incubated with MTT $(0.5 \mathrm{mg} / \mathrm{mL}$ in cell culture medium $)$ for $2 \mathrm{~h}$.
The formazan crystals were dissolved with dimethyl sulfoxide (DMSO; Sigma-Aldrich Co.). Absorbance of the extracted solubilized formazan was measured at $570 \mathrm{~nm}$ by subtracting the background signal in the 96-well plate with a multimode microplate reader (Synergy HT; BioTek Instruments, Inc.).

\section{Cellular uptake of Au nanostructures and their intracellular distribution}

To quantify the cellular uptake of Au nanostructures, Au NRs and $\mathrm{Au}$ NPs were respectively suspended in serum-free cell culture medium at the same gold concentration $(40 \mu \mathrm{M})$ and added into cultured MDA-MB-231 cells with identical density. Upon incubation for $4 \mathrm{~h}$, free particles were removed by washing thoroughly with Hank's buffered salt solution (HBSS) at least three times. Then, the cells were trypsinized, centrifuged, and washed again with HBSS before collection. The collected cells were lysed with an alkaline buffer consisting of sodium dodecyl sulfate and $1 \mathrm{~N}$ sodium hydroxide and the lysates were analyzed by spectrometric assay for Au nanostructure quantification as described previously. ${ }^{16}$ The normalization was done via the areas under the UV absorbance spectrum (400-800 nm); that is, the area was calculated in the untreated control group and then subtracted from that in Au NP and Au NR groups respectively. Then, the value obtained in the Au NR group was divided by the value in the Au NP group for normalization.

To determine the intracellular destination of different $\mathrm{Au}$ nanostructures after uptake, MDA-MB-231 cells incubated with respective $\mathrm{Au}$ nanostructures as described above were rinsed with HBSS to remove free particles, trypsinized, washed, and centrifuged into cell pellets. Cell pellets were fixed with $4 \%$ paraformaldehyde (EM Sciences, Hatfield, PA, USA) for $2 \mathrm{~h}$ at room temperature, postfixed with $1 \%$ osmium tetraoxide for $1 \mathrm{~h}$, dehydrated in a graded series of ethanol solutions, and then embedded in epoxy resin. Ultrathin sections $(\sim 70 \mathrm{~nm})$ were obtained by a Leica Ultracut Ultramicrotome (Leica Microsystems Inc., Buffalo Grove, IL, USA) and examined with a TEM (Philips CM20; FEI). Monolayer cells cultured on a well plate and treated with different Au nanostructures were also fixed and embedded as above. Then, the Au nanostructure-incubated cells were examined under a focused ion beam scanning electron microscope (Zeiss Auriga Small Dual-Beam FIBSEM; Carl Zeiss Microscopy, LLC., Thornwood, NY, USA).

\section{Intracellular ${ }^{\mathrm{O}} \mathrm{O}_{2}$ measurement}

For the cell-based intracellular ${ }^{1} \mathrm{O}_{2}$ measurement, SOSG was prepared at a final concentration of $1.33 \mu \mathrm{M}$ in HBSS according to the manufacturer's protocol. MDA-MB-231 cells treated with 5-ALA with/without Au nanostructures were incubated with the SOSG solution for $2 \mathrm{~h}$ and refreshed with 
$100 \mu \mathrm{L}$ HBSS for light irradiation. After irradiation, the cells were further incubated at $37^{\circ} \mathrm{C}$ in the dark for $20 \mathrm{~min}$ and then the fluorescence intensity was measured using the microplate reader with the excitation wavelength at $485 / 20 \mathrm{~nm}$ and the emission wavelength at 528/20 nm.

\section{Cellular photodynamic therapy}

The PDT procedure was conducted according to our previous studies. ${ }^{15,22}$ Briefly, cells were seeded into a 96-well plate at a concentration of $3 \times 10^{4}$ cells $/ \mathrm{mL}$ in $200 \mu \mathrm{L}$ culture medium, and cultured for $24 \mathrm{~h}$ prior to PDT treatment. The final cell density is 6,000 cells per well in the 96-well plate, which is within the range of $5 \times 10^{2}-5 \times 10^{5}$ cells per well for the following MTT assay according to the protocol. Afterwards, the culture medium was then replaced with the serum-free medium containing 5-ALA with/without different $\mathrm{Au}$ nanostructures. The final concentrations of gold and 5-ALA in all experiments were kept at $40 \mu \mathrm{M}$ and $1 \mathrm{mM}$, respectively. Serum-free medium was used as blank controls. After incubation for $4 \mathrm{~h}$ in dark, the media were replaced with $100 \mu \mathrm{L}$ HBSS and the cells were irradiated from the top with a broadband light source using a $150 \mathrm{~W}$ halogen lamp $\left(100 \mathrm{~mW} / \mathrm{cm}^{2}\right.$, Dolan-Jenner Fiber-Lite MI-150; Dolan-Jenner Industries, Boxborough, MA, USA) for $1 \mathrm{~min}$. For the NIR light irradiation, the same light source but filtered through $600 \mathrm{~nm}$ or $700 \mathrm{~nm}$ filters (FEL600 and FEL 700; Thorlabs, Inc., Newton, NJ, USA) was applied to cells for 10, 20, and $30 \mathrm{~min}$, respectively. After irradiation, the culture was replaced with complete culture media and incubated for $24 \mathrm{~h}$ prior to any further evaluations.

\section{Cell viability assessment}

The cell viability upon different treatments was determined by MTT assay as described in the cytotoxicity test above. To confirm the MTT assay results, MDA-MB-231 cells after PDT treatment were also fluorescently stained for viability using a Live/Dead VR Viability/Cytotoxicity kit (Invitrogen, Thermo Fisher Scientific, Waltham, MA, USA). Briefly, cells cultured on glass cover slips after PDT treatment were gently washed with HBSS and then incubated with the Live/Dead VR Viability/Cytotoxicity solution for $30 \mathrm{~min}$. Viable cells were stained green by calcein acetoxymethyl $(0.05 \%)$, while the nuclei of dead cells were stained red by ethidium homodimer-1 $(0.2 \%)$. The stained cells were examined under a Nikon Eclipse 80i epifluorescent microscope (Nikon Instruments, Melville, NY, USA). To visualize cell morphology changes after PDT treatment, cultures were fixed with $4 \%$ formalin and stained with phalloidin (Alexa FluorH 488 phalloidin;
Biotium Inc, Hayward, CA, USA) for filamentous actin and 4,6-diamidino-2-phenylindole (dilactate) for cell nuclei. The stained cells were also examined under the Nikon Eclipse 80i epifluorescent microscope.

\section{Statistical analysis}

All quantitative results were obtained from at least triplicate samples. Data were expressed as the mean \pm SD. Unpaired Student's $t$-test was used in the statistical analysis of experimental data. $p<0.05$ was considered statistically significant.

\section{Results and discussion Characterization of Au nanostructures}

Following a similar synthesis procedure as described previously, ${ }^{23}$ colloidal Au NRs were facilely prepared by galvanic replacement of sacrificial cobalt nanoparticles in gold salt solution with low molecular weight PVP as a stabilizing agent. In contrast to the solid spherical structures of $\mathrm{Au}$ NPs, the as-prepared Au NRs exhibited a typical ringlike structure (Figure 1A). Depending on the size of cobalt nanoparticles, $\mathrm{Au} / \mathrm{Co}$ ratio, and PVP molecular weight, the outer diameter, inner diameter, and height of Au NRs could be tuned. ${ }^{23}$ For this study, the outer diameter of Au NRs was kept similar to the diameter of Au NPs with an average of $\sim 40 \mathrm{~nm}$ as determined by dynamic light scattering measurement. The Polydispersity Index for Au NPs and Au NRs is 0.264 and 0.272 , respectively. The exact geometry parameters of $\mathrm{Au}$ nanostructures are schematically illustrated in Figure 1. Although PVP was used as the stabilizing agent for both Au NRs and Au NPs, a slightly negative zeta potential $(-10 \mathrm{mV})$ was measured with Au NPs, while Au NRs remained electrically neutral $(0 \mathrm{mV})$. This may come from the short chain of PVP that cannot completely shield the negatively charged citrate. Due to structural difference, $\mathrm{Au}$ NRs showed distinctive SPR properties from Au NPs. As shown in Figure 1B, Au NPs had a maximum absorbance wavelength at $\sim 528 \mathrm{~nm}$ in the UV-visible absorption spectra, while Au NRs showed a red shift of the SPR peak with a maximum absorbance wavelength at $653 \mathrm{~nm}$.

\section{Simulation of optical spectra and near- field distribution of Au nanostructures}

As shown in Figure 1C, the calculated extinction peak at $510 \mathrm{~nm}$ for $\mathrm{Au}$ NPs and $690 \mathrm{~nm}$ for Au NRs through DDA simulation is the net result of absorption and scattering by Au nanostructures, which is in satisfactory agreement with experimental results. Note that a broadening of the SPR band and a slight shift of the peak position were observed in the experimental spectra for both $\mathrm{Au}$ NPs and Au NRs. 

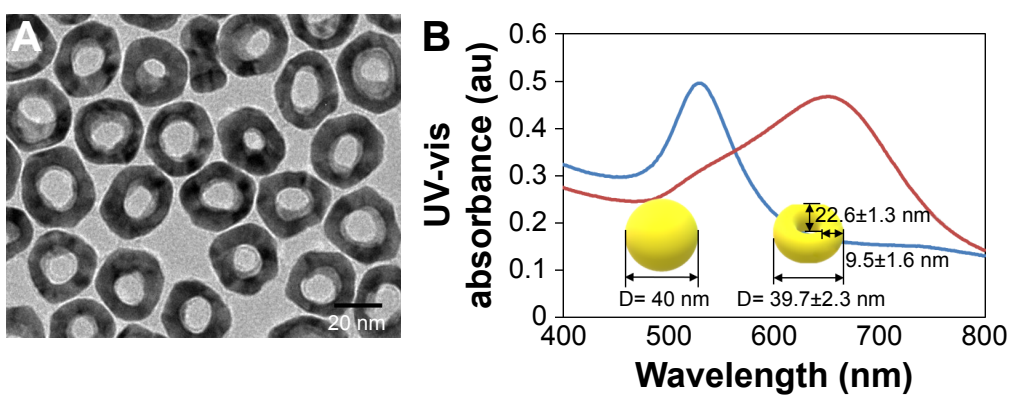

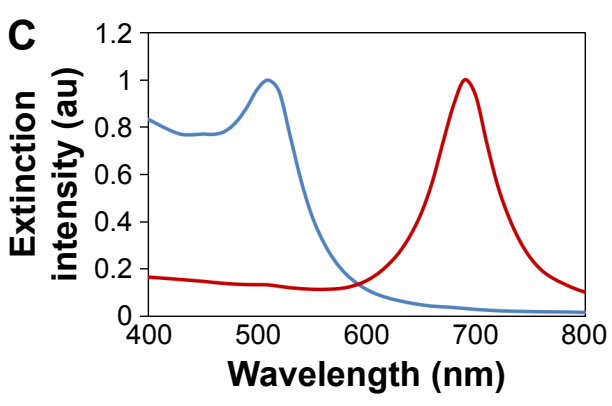

D
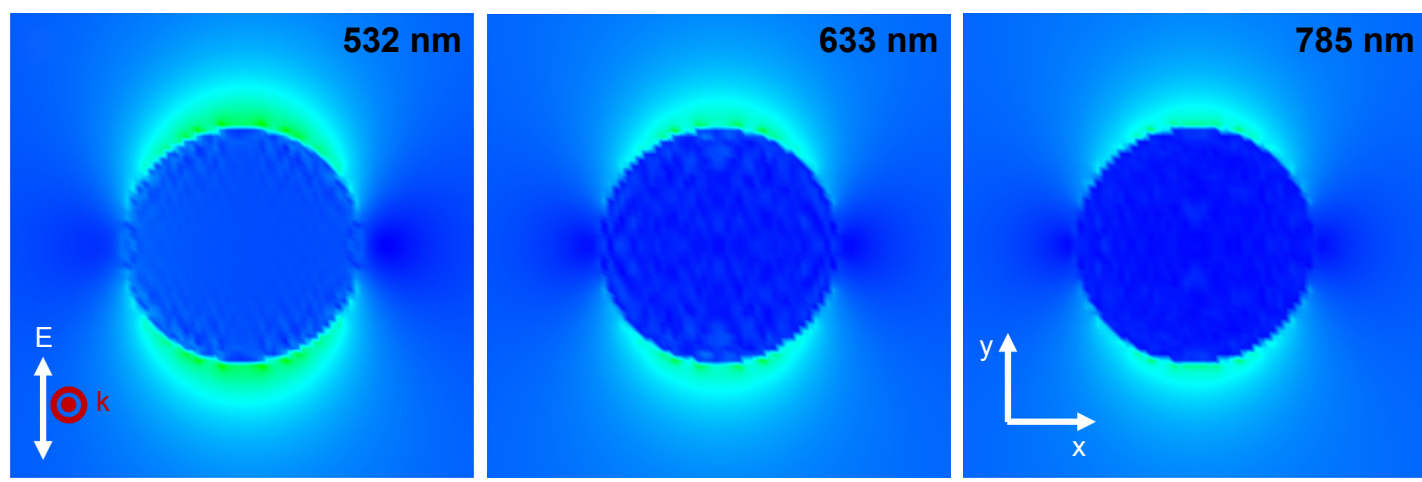

Intensity
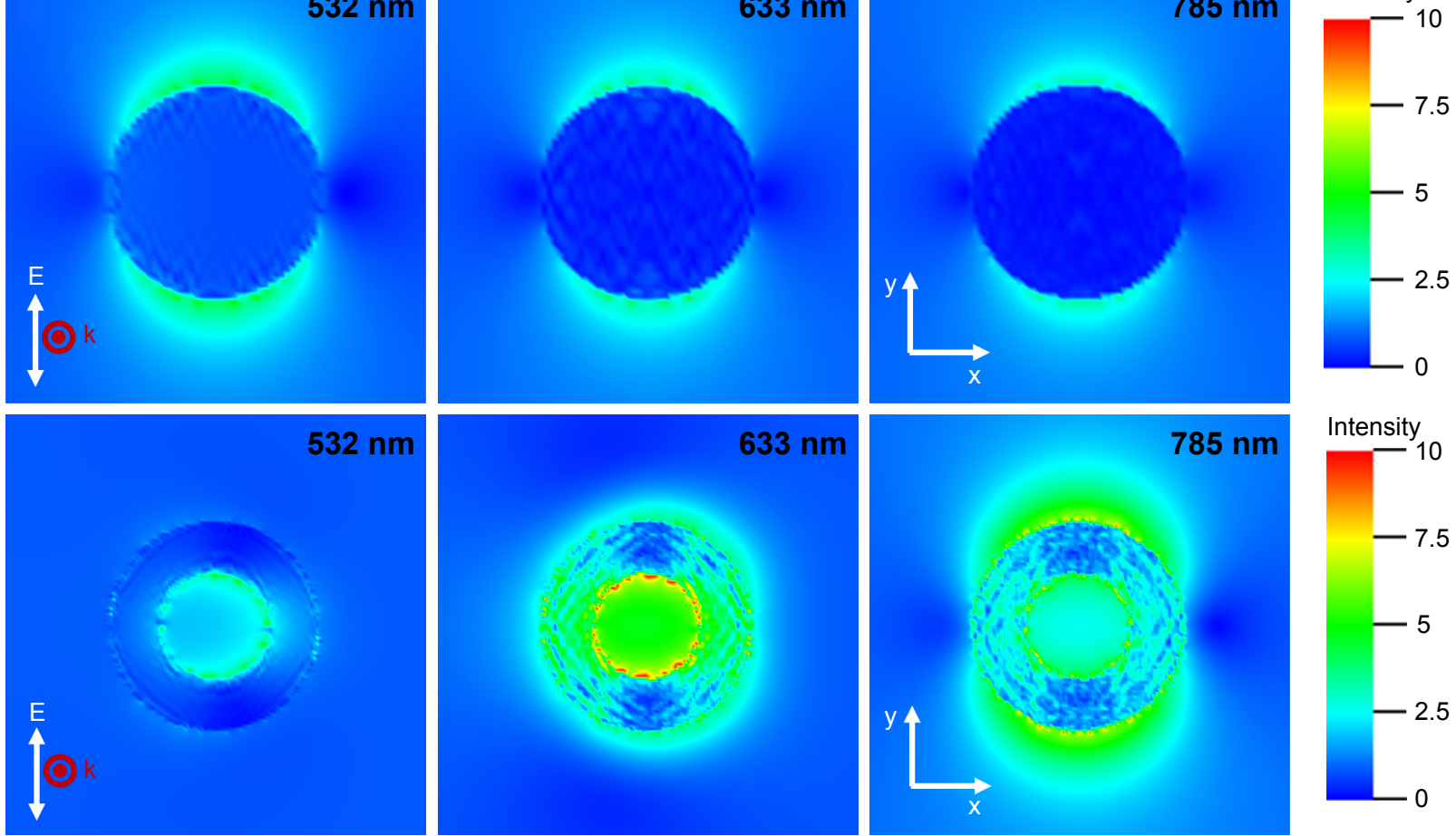

Figure I (A) Transmission electron microscope micrographs of Au NRs. (B) UV-vis absorption spectra of Au NPs and Au NRs. (C) Simulated extinction spectra of Au NPs and Au NRs through DDA calculation. (D) DDA simulated electric field intensity distribution of Au NPs (upper row) and Au NRs (lower row) at 532 nm, 633 nm, and $785 \mathrm{~nm}$ wavelength, respectively.

Notes: The red point and the white arrow are the propagation direction ( $\mathrm{k}$ vector) and the polarization (E field vector), respectively. The color bar represents the value of |E|. The coordinate directions are as indicated.

Abbreviations: Au NP, gold nanoparticle; Au NR, gold nanoring; DDA, discrete dipole approximation; UV-vis, ultraviolet-visible.

This could most likely be attributed to size variation and interparticle distributions in as-prepared gold nanomaterials. Clearly, the morphology of Au nanostructures has a strong influence on their interactions with light, resulting in a noticeable red shift of the resonance peak towards the NIR region in the case of Au NRs. Due to the presence of SPR, the intensity of the localized EM field near the surface of $\mathrm{Au}$ nanostructures would also closely correlate with their morphology. To better understand the differential enhancement of the EM field by Au NPs and Au NRs, theoretical calculation was done using DDA methods. The respective simulation images of EM field distribution for Au NPs and $\mathrm{Au}$ NRs at excitation light with different wavelengths
(532 nm, $633 \mathrm{~nm}$, and $785 \mathrm{~nm}$ ) are depicted in Figure 1D. The calculated maximum electric field ratio between Au NPs and Au NRs at each wavelength is 1:1.5, 1:6.4, and 1:5.4, respectively. In consistence with our previous report on the simulation using finite-difference time domain modeling, ${ }^{23}$ the EM field inside the Au NR cavity was substantially enhanced, which is probably attributed to the significant coupling between the two EM modes of the inner and outer surfaces of the ring structure. Comparison of the EM field enhancement under incident light with various wavelengths shows the maximum field enhancement at $532 \mathrm{~nm}$ for Au NPs and $633 \mathrm{~nm}$ for Au NRs, suggesting shape-dependent LSPR of Au nanostructures. 


\section{Enhanced ' $\mathrm{O}_{2}$ generation induced by $\mathrm{Au}$ nanostructure-PS}

To determine the contribution of the plasmonic effect of $\mathrm{Au}$ nanostructures to ${ }^{1} \mathrm{O}_{2}$ formation as well as to ascertain the shape-dependent ${ }^{1} \mathrm{O}_{2}$ enhancement, PpIX, a derivative of 5-ALA, was used as a model PS. PpIX as a commercial PDT drug has shown good success against skin basal cell carcinoma and squamous cell carcinoma. ${ }^{30}$ Using SOSG as the highly selective fluorescent probe for ${ }^{1} \mathrm{O}_{2},{ }^{31}$ we were able to monitor the time-resolved formation of ${ }^{1} \mathrm{O}_{2}$ from PpIX with the presence of Au nanostructures. By offsetting the contribution of SOSG, Figure 2 shows the time-resolved net ${ }^{1} \mathrm{O}_{2}$ formation enhanced by Au NPs and Au NRs with the presence of PpIX under various light irradiations. Based on Figure 2A, we calculated the ${ }^{1} \mathrm{O}_{2}$ enhancement ratio of 1:1.43 between Au NP-PpIX and Au NR-PpIX under the broadband light irradiation. To determine whether such an enhancement would remain for longer wavelengths, especially in the NIR range, broadband light respectively filtered through $600 \mathrm{~nm}$ and $700 \mathrm{~nm}$ LP filters was used as the irradiation source. Clearly, the Au NR-PpIX-elevated ${ }^{1} \mathrm{O}_{2}$ generation was continuously observed (Figure 2B and 2C). As calculated, the enhancement ratio between Au NP-PpIX and Au NR-PpIX for ${ }^{1} \mathrm{O}_{2}$ generation with $600 \mathrm{~nm}$ LP filtered light was 1:1.34 after 20 min irradiation and with $700 \mathrm{~nm}$ LP filtered light it was 1:1.20. Apparently, these results affirm the shape dependence of Au nanostructure-assisted ${ }^{1} \mathrm{O}_{2}$ generation, which to a certain degree agrees well with the EM field distribution from the theoretical simulation (Figure 1D). Despite their similar physicochemical characteristics (ie, surface chemistry and size) to solid Au NPs, Au NRs did demonstrate a much higher efficiency in promoting ${ }^{1} \mathrm{O}_{2}$ formation. It is speculated that when the incident light is in resonance with the SPR of $\mathrm{Au}$ NRs, a substantial enhancement of the EM field would occur in the ring cavity besides the inner and outer surfaces, which cannot be seen with solid Au NPs. With a comparable surface area, easy access of PpIX to the central region of Au NRs would lead to more activation of PpIX and consequently more ${ }^{1} \mathrm{O}_{2}$ formation than that of Au NPs.

As noted in Figure 1, the SPR absorbance of Au NRs is in the NIR range, thus, it would be of great benefit to achieve enhanced ${ }^{1} \mathrm{O}_{2}$ generation under light irradiation with longer wavelengths $(\lambda>600 \mathrm{~nm})$. For efficient photodynamic reaction, matching the PS absorption with excited light spectra becomes crucial
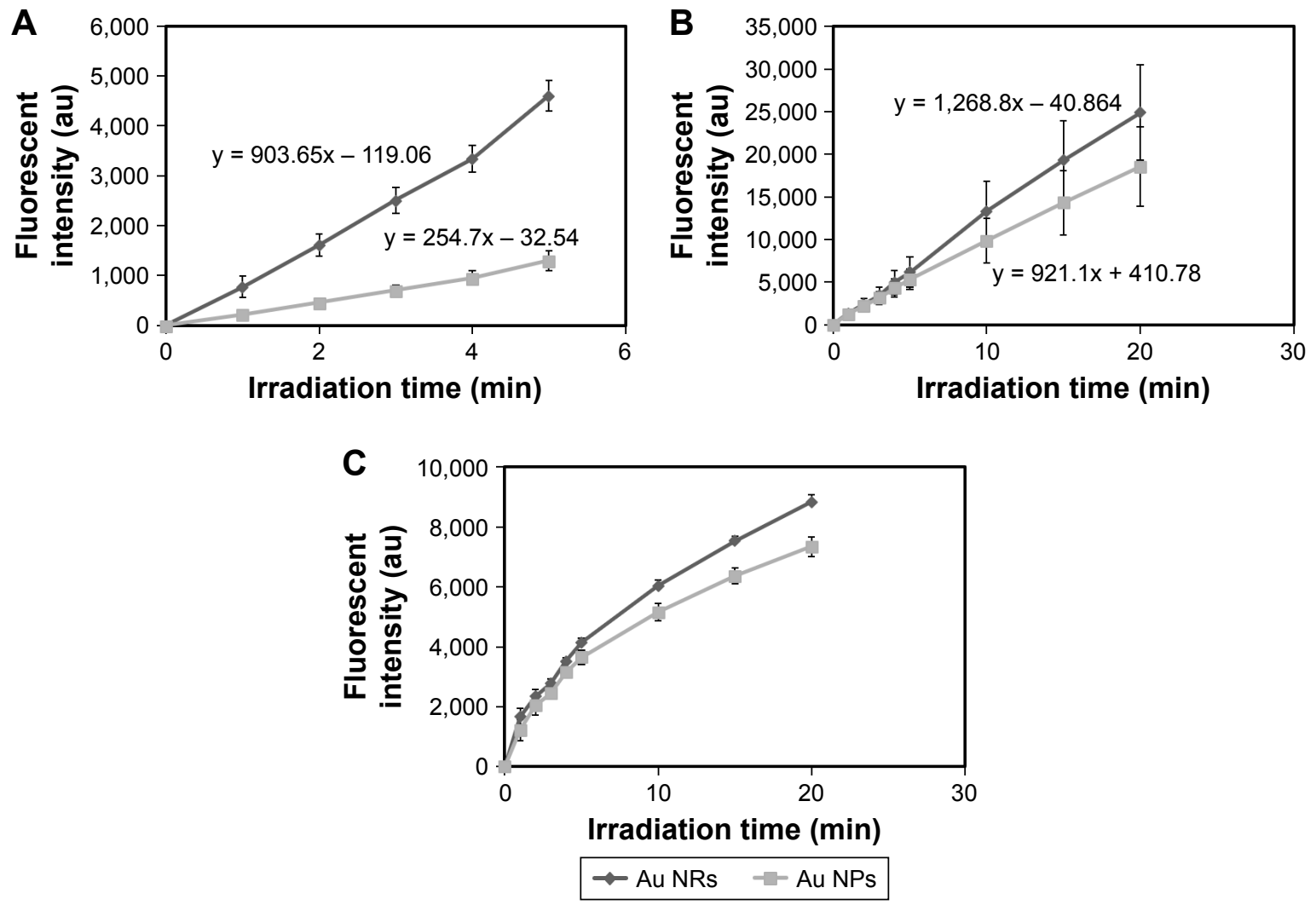

Figure 2 Time-resolved kinetics of single oxygen formation from protoporphyrin IX in the presence of Au NPs and Au NRs under irradiation with (A) broadband light, (B) $600 \mathrm{~nm}$ LP filtered light, and (C) $700 \mathrm{~nm}$ LP filtered light.

Notes: Singlet oxygen was measured using the singlet oxygen sensor green reagent. The data presented were obtained from three independent experiments.

Abbreviations: Au NP, gold nanoparticle; Au NR, gold nanoring. 
for maximum quantum yield of ${ }^{1} \mathrm{O}_{2}$. As PpIX itself cannot be irradiated efficiently under the NIR light, we thus postulate that the SPR peak of Au NRs could, at least partially, fit with $600 \mathrm{~nm}$ LP and $700 \mathrm{~nm}$ LP filtered light to induce an increase of the photonic energy upon light irradiation and subsequently enhance the photocurrent between Au NRs and PpIX. Through nonradiative energy transfer and coupling to the surface plasmons of Au NRs, more PpIX can be excited to their triplet and singlet states and therefore result in more ${ }^{1} \mathrm{O}_{2}$ generation (Figure $2 \mathrm{~B}$ and $2 \mathrm{C}$ ).

\section{Cytotoxicity and cellular uptake of Au nanostructures}

Biocompatibility of various Au nanostructures was examined prior to following cell-based PDT tests. As shown in Figure 3A,
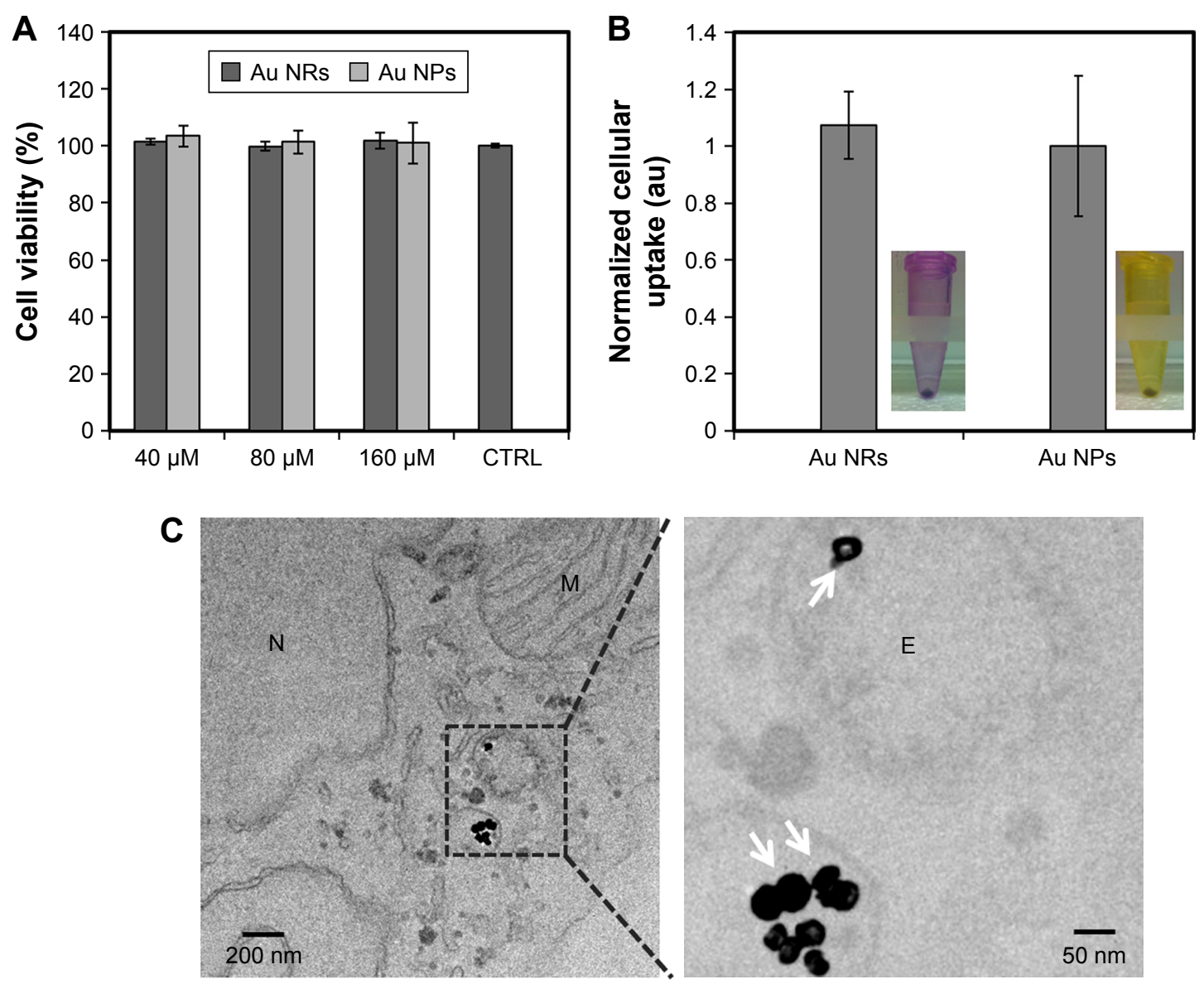

D
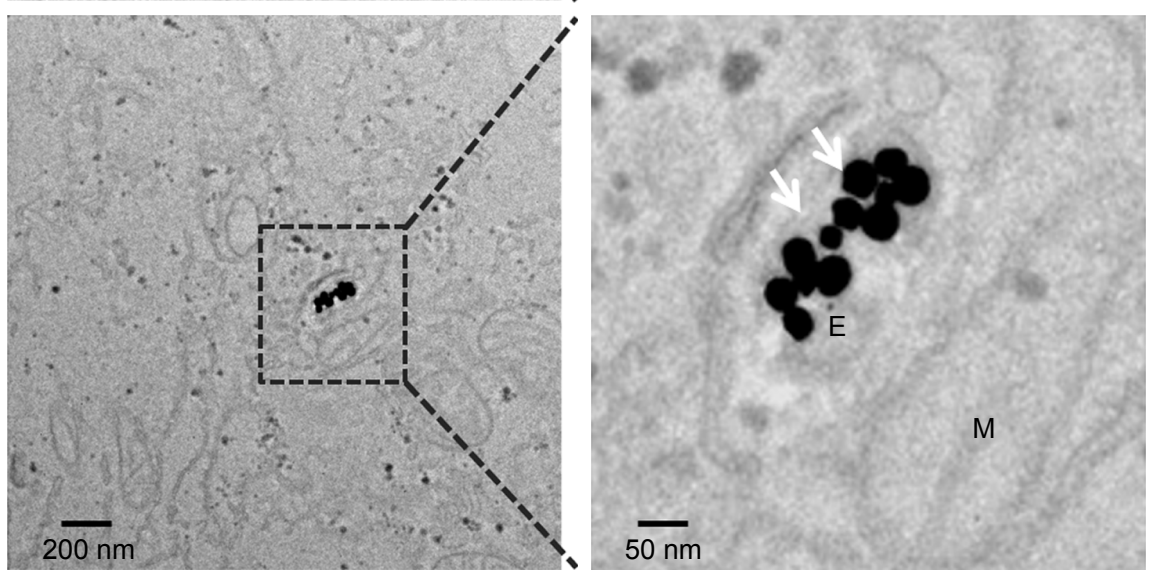

Figure 3 (A) Biocompatibility of Au NRs and Au NPs, in which MDA-MB-23I cells were incubated with Au NRs and Au NPs at concentrations of 40, 80 , and I60 $\mu$ M for $24 \mathrm{~h}$ and then viable cells were determined by MTT assay. (B) Uptake of different Au nanostructures after incubation for $4 \mathrm{~h}$ with MDA-MB-23। cells. The collected cells were lysed and the lysates were analyzed by spectrometric assay for gold nanostructure quantification. The normalization was done via the areas under the ultraviolet absorbance spectrum (400-800 nm); that is, the area was calculated in the Au NP and Au NR groups and then subtracted from the untreated control group. Then, the value obtained in the Au NR group was divided by the value in the Au NP group for normalization. Transmission electron microscope micrographs of the cells incubated with (C) Au NRs and (D) Au NPs for $4 \mathrm{~h}$. Intracellular Au NPs indicated by white arrows.

Abbreviations: Au NP, gold nanoparticle; Au NR, gold nanoring; CTRL, control; E, endosome; M, mitochondrion; N, nucleus. 
both Au nanostructures at various concentrations showed negligible cytotoxicity to MDA-MB-231 cells after 24-h incubation. As the amount of Au nanostructures ingested by cells would contribute to the treatment efficiency of PDT, cellular uptake of different $\mathrm{Au}$ nanostructures was also evaluated for the possible dependence on morphology. Upon complete release from the collected cells, the amount of $\mathrm{Au}$ nanostructures ingested by MDA-MB-231 cells was quantified spectrometrically and no significant difference was observed between $\mathrm{Au}$ NPs and Au NRs, indicating that the morphological change of Au NRs may have a minimal effect on the cellular uptake (Figure 3B). Although Au NRs have distinct morphology from solid Au NPs, they both share the circular shape with a similar outer diameter. As evidenced from previous studies, the diameter of Au spheres plays an essential role in regulating their cellular endocytosis and exocytosis and the size of $40-50 \mathrm{~nm}$ seems optimal for intracellular accumulation. ${ }^{32}$ Thus, it is not surprising to see comparable accumulation of Au NPs and Au NRs in MDA-MB-231 cells.

To further understand the intracellular distribution of different $\mathrm{Au}$ nanostructures, thin slices of the cell pellets posttreated with respective Au nanostructures were examined under a TEM. Regardless of particle morphology, a majority of Au nanostructures was located in the intracellular vesicles (Figure 3C and 3D). Direct visualization of the cells incubated with Au NRs under a bright-field confocal microscope and the focused ion beam scanning electron microscope examination of the cell cross-sections also confirmed the cellular internalization of Au NRs (see Figure S1). Clearly, minimal cytotoxicity and efficient uptake by breast cancer cells imply the potential of Au NRs in cancer PDT.

\section{Enhanced intracellular ${ }^{\prime} \mathrm{O}_{2}$ generation enabled by Au nanostructure-PS}

Intracellular elevation of ${ }^{1} \mathrm{O}_{2}$ production upon light irradiation is a crucial indicator for enhancing the PDT efficacy. ${ }^{33}$ Intracellular ${ }^{1} \mathrm{O}_{2}$ formation and corresponding cellular destruction following PDT under various conditions are indicated in Figure 4. Following a similar trend to that of the cell-free study on ${ }^{1} \mathrm{O}_{2}$ formation, the combination of $\mathrm{Au}$ NRs and 5-ALA (Au NRs+5-ALA) yielded the highest intracellular ${ }^{1} \mathrm{O}_{2}$ level after broadband light irradiation (Figure 4A). More importantly, upon irradiation with a longer wavelength (ie, $600 \mathrm{~nm} \mathrm{LP}$ and $700 \mathrm{~nm}$ LP filtered light), cells treated with 5-ALA and Au NRs still yielded the highest ${ }^{1} \mathrm{O}_{2}$, significantly higher than the other two groups (Figure 5A and 5B). To ensure the increase of intracellular ${ }^{1} \mathrm{O}_{2}$ is not from the increase of intracellular accumulation of PS, we quantified the intracellular PS (ie, PpIX) by measuring the fluorescence intensity. Interestingly, no significant difference was identified among all three groups (data not shown). Thus, it is believed that Au nanostructures have minimum effect on the cellular uptake of 5-ALA, or at least the production rate of PpIX, and they may mainly work as the ${ }^{1} \mathrm{O}_{2}$ enhancer in the present study. Consistent with cell-free conditions, the intensified EM fields in the vicinity of excited intracellular Au nanostructures can activate PS via resonance energy transfer, leading to a higher level of ${ }^{1} \mathrm{O}_{2}$ generation. Once again, the significant difference in intracellular ${ }^{1} \mathrm{O}_{2}$ levels found between Au NRs and Au NPs is primarily attributed to their structural distinction; that is, the substantially enhanced EM field of Au NRs would activate more PS trapped in the cavity.

To our interest, under NIR light irradiation, the highest ${ }^{1} \mathrm{O}_{2}$ level was consistently observed in the cells treated with $\mathrm{Au}$ NRs+5-ALA, and discernible in those with Au NPs+5ALA or 5-ALA alone (Figure 5A and 5B). It is noted that intracellular PpIX alone (maximum absorbance at $\sim 400 \mathrm{~nm}$ ) could be barely activated by the NIR light, ${ }^{34}$ as also was seen for the solid Au NPs with a characteristic SPR peak at $\sim 530 \mathrm{~nm}$. On the contrary, Au NRs with resonant absorption at a NIR wavelength will produce an intensified EM field and sufficient energy transfer for PS activation and ${ }^{1} \mathrm{O}_{2}$ generation. Therefore, great intracellular ${ }^{1} \mathrm{O}_{2}$ enhancement can still be achieved with the presence of Au NRs with strong SPR absorption under NIR light irradiation.

\section{Au nanostructure-PS assisted cellular PDT}

The efficacy of elevated ${ }^{1} \mathrm{O}_{2}$ in killing cancer cells with the presence of different Au nanostructure-PS combinations was determined by MTT assay. As shown in Figure 4B, under broadband light irradiation for $1 \mathrm{~min}$, both Au NRs and Au NPs could lead to more cell killing than 5-ALA only. In particular, Au NRs +5-ALA yielded the highest destruction of MDA-MB231 cells $(75.5 \%)$, accounting for about twofold that from 5-ALA alone (33.9\%) and 1.5 times that from a 5-ALA+Au NP combination. This observation was confirmed by fluorescently staining MDA-MB-231 cells for viability (live/dead staining) and morphology (filamentous actin staining) after various PDT treatments. Consistent with MTT results, more dead cells were found in the Au NRs+5-ALA group (Figure 4C and 4D). Considering negligible cytotoxicity and comparable cellular uptake of both Au NRs and Au NPs, the improved cell killing by Au NRs must be from its structural contribution, offering unique SPR properties and a significant increase of intracellular ${ }^{1} \mathrm{O}_{2}$ 

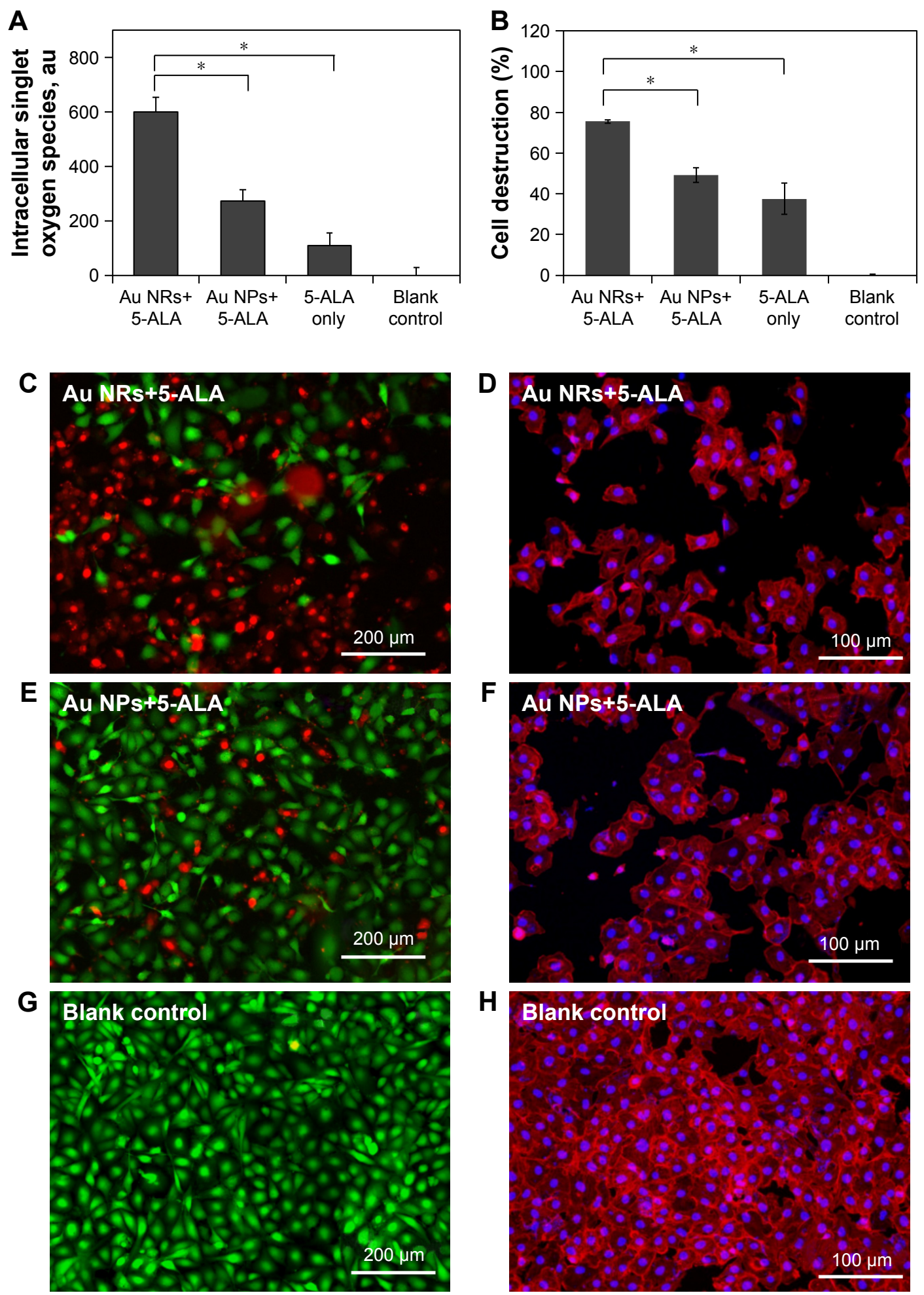

Figure 4 (A) Elevated generation of singlet oxygen in MDA-MB-23। cells upon various PDT treatments with broadband light irradiation for I min. (B) Cell destruction rate of MDA-MB-23I cells after various PDT treatments with broadband light irradiation for I min. Representative fluorescent images of MDA-MB-23। cells stained with a live/dead kit (left column, live cells stained green with calcein acetoxymethyl and dead cells stained red with ethidium homodimer-I), and sulforhodamine I0I-conjugated phalloidin (right column, intracellular filamentous actin stained red and cell nuclei stained blue with DAPI) after various PDT treatments under broadband light irradiation for I min. (C) and (D) Au NRs+5-ALA, (E) and (F) Au NPs+5-ALA, (G) and (H) blank controls (no 5-ALA and Au nanostructures).

Notes: Prior to light irradiation, cells were treated with the combination of Au NRs and 5-ALA (Au NR+5-ALA), the combination of Au NPs and 5-ALA (Au NPs+5-ALA), and 5-ALA alone without Au nanostructures in serum-free medium. The final concentration of gold and 5-ALA as maintained at $40 \mu M$ and I mM for each group. Cells treated with serum-free medium without Au nanostructures or 5-ALA were considered as blank control. The data are representative of three separate experiments; $* p<0.0$ I. Abbreviations: 5-ALA, 5-aminolevulinic acid; Au NP, gold nanoparticle; Au NR, gold nanoring; PDT, photodynamic therapy. 
A
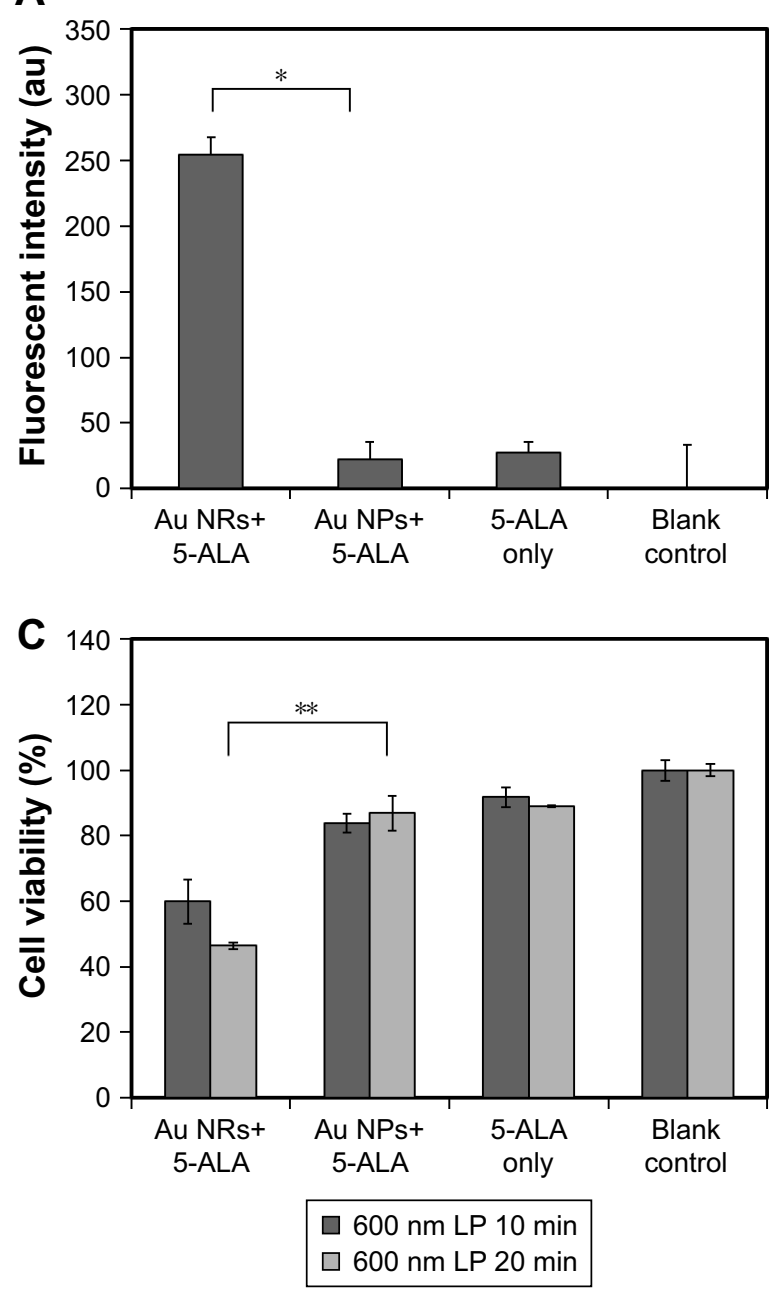

B
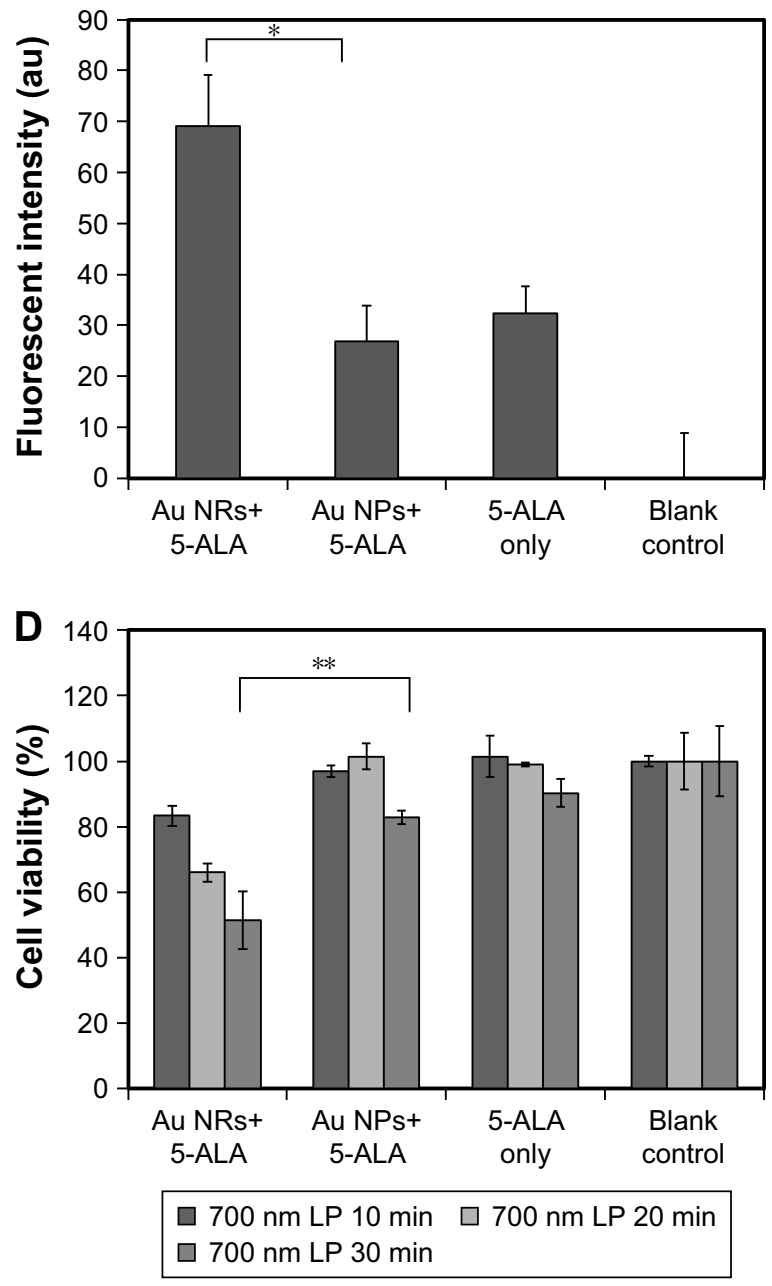

Figure 5 (A) Elevated generation of singlet oxygen in MDA-MB-23। cells after PDT treatments with $600 \mathrm{~nm}$ LP filtered light for 20 min and (B) $700 \mathrm{~nm}$ LP filtered light for $30 \mathrm{~min}$. The data are representative of three separate experiments; ${ }^{*} p<0.01$. Cell viability of MDA-MB-23I cells after PDT treatment under (C) $600 \mathrm{~nm}$ LP filtered light for 10 and $20 \mathrm{~min}$ and (D) $700 \mathrm{~nm}$ LP filtered light irradiation for 10, 20, and $30 \mathrm{~min}$. Cell viability was determined by MTT assay $24 \mathrm{~h}$ after treatment. **p $<0.05$.

Notes: Prior to light irradiation, cells were treated with the combination of Au NRs and 5-ALA (Au NRs+5-ALA), the combination of Au NPs and 5-ALA (Au NPs+5-ALA), and 5-ALA alone (5-ALA only) without Au nanostructures in serum-free medium. The final concentration of gold and 5-ALA was maintained at $40 \mu M$ and I mM for each group. Cells treated with serum-free medium without Au nanostructures or 5-ALA were considered as blank control.

Abbreviations: 5-ALA, 5-aminolevulinic acid; Au NP, gold nanoparticle; Au NR, gold nanoring; PDT, photodynamic therapy.

generation. Note that the 5-ALA-Au nanostructure-induced cell destruction became less proportional to the elevated levels of ${ }^{1} \mathrm{O}_{2}$, which may be due to the intrinsic intracellular protection mechanism against ${ }^{1} \mathrm{O}_{2}{ }^{35}$ Depending on the dosage and location of ${ }^{1} \mathrm{O}_{2}$ formation, ${ }^{1} \mathrm{O}_{2}$-triggered cell killing comprises apoptosis or apoptosis in combination with necrosis, involving multiple signaling pathways, and is further complicated by the anti-apoptotic and pro-apoptotic regulation of the Bcl-2 family in mitochondria. ${ }^{36-38}$

Similar to the findings of cell-free ${ }^{1} \mathrm{O}_{2}$ generation, under NIR light irradiation only Au NRs+5-ALA treatment yielded noticeable cell destruction but not the other two groups (Figure 5C and 5D). With either $600 \mathrm{~nm}$ LP filtered light for $20 \mathrm{~min}$ or $700 \mathrm{~nm}$ LP filtered light for $30 \mathrm{~min}$
Au NRs+5-ALA led to $40.5 \%$ or $31.4 \%$ more cell killing than Au NPs+5-ALA, respectively. Consistently with numerical simulation, the concentrated EM field near Au NRs under NIR light exposure may play a key role resulting in an increased population of excited PSs. Meanwhile, photobleaching of PSs could be affected by their easy entry to the center cavity of Au NRs, and more excitation-emission cycles would occur prior to photobleaching of PSs during the excited state; that is, PSs can be activated and generate singlet oxygen more efficiently. ${ }^{39}$ This finding becomes clinically relevant especially considering that NIR light normally has better tissue penetration depth. ${ }^{40}$ The use of NIR light for PDT can potentially address the major technical challenge associated with current PDT practice; that is, it is only suitable for superficial tumor 
tissues due to limited light penetration. The SPR properties of $\mathrm{Au}$ NRs in the NIR region hold particular advantages for extending current PDT towards deep tumor treatment.

Both $\mathrm{Au}$ NPs and Au NRs have the potential to convert photonic energy into heat especially under intensive light irradiation, which accounts for the photothermal effects as reported elsewhere. ${ }^{41,42}$ However, it was found that tumor cells usually required relatively high laser energy to be photothermally damaged even with the assistance of $\mathrm{Au}$ nanostructures. ${ }^{42}$ The energy density of the irradiation source in our study $\left(100 \mathrm{~mW} / \mathrm{cm}^{2}\right)$ is at least 10 times lower than that employed in photothermal therapy. Therefore, cell destruction caused by the temperature changes due to photothermal effects can be excluded from our study. To affirm this, separate control experiments were performed to compare the cell viability of cells treated with Au nanostructures alone under light irradiation. Negligible cell destruction was detected for both 20-min and 30-min irradiation with $700 \mathrm{~nm}$ LP filtered light (Figure S2). In addition, early reports also suggested that the generation of ${ }^{1} \mathrm{O}_{2}$ can be induced directly by noble metal nanostructures under light irradiation. ${ }^{43-46}$ However, the formation of ${ }^{1} \mathrm{O}_{2}$ caused by $\mathrm{Au}$ nanostructures alone has also demonstrated great dependence on the dosage and energy density of the irradiating light source. Pulsed lasers rather than a continuous wavelength light source, which was used in the current study, would result in much more significant ${ }^{1} \mathrm{O}_{2}$ generation relying on Au nanostructures themselves under light exposure. ${ }^{43}$ We have also confirmed that Au NPs alone did not cause significant enhanced ${ }^{1} \mathrm{O}_{2}$ generation with or without light irradiation (data not shown). Therefore, the combinative effects of $\mathrm{Au}$ nanostructures and PSs should be the major contributor to enhanced intracellular ${ }^{1} \mathrm{O}_{2}$ and subsequent increased cell death in our present findings.

\section{Conclusions}

In this study, shape-dependent SPR properties of different $\mathrm{Au}$ nanostructures were characterized and studied for their performance under various PDT conditions. As a result of the unique morphological attributes, Au NRs exhibited plasmonic resonance in the NIR range, exhibiting efficient PS activation and corresponding ${ }^{1} \mathrm{O}_{2}$ generation for cell destruction with NIR light irradiation. Clearly, cell destruction enabled by NIR-absorbing $\mathrm{Au}$ NRs sheds promising light on the efforts to address the depth limitation with current PDT for deep tumor therapy. Although not fully explored, the interior space and outer surface of Au NRs can be separately modified for designated functionality, which offers multifaceted potentials in chemical and biological sensing, drug delivery, and bioimaging.

\section{Acknowledgments}

This work was supported by the Natural Science Foundation of China (Grant Number: 81601532 and 31428007 ), the Natural Science Foundation of Jiangsu Province (BK20160814), and the Scientific Research Foundation of Nanjing University of Aeronautics and Astronautics (1003-YAH16009). Due to a recent reorganization, the affiliation for Dr Hongjun Wang is now known as Department of Biomedical Engineering, Stevens Institute of Technology, Hoboken, NJ, USA.

\section{Disclosure}

The authors report no conflicts of interest in this work.

\section{References}

1. Kim EY, Kumar D, Khang G, Lim D-K. Recent advances in gold nanoparticle-based bioengineering applications. J Mater Chem B. 2015; 3(43):8433-8444.

2. Huang X, El-Sayed MA. Gold nanoparticles: Optical properties and implementations in cancer diagnosis and photothermal therapy. J Adv Res. 2010;1(1):13-28.

3. Willets KA, Van Duyne RP. Localized surface plasmon resonance spectroscopy and sensing. Annu Rev Phys Chem. 2007;58(1):267-297.

4. Messersmith RE, Nusz GJ, Reed SM. Using the localized surface plasmon resonance of gold nanoparticles to monitor lipid membrane assembly and protein binding. J Phys Chem C Nanomater Interfaces. 2013; 117(50):26725-26733.

5. Zong J, Cobb SL, Cameron NR. Peptide-functionalized gold nanoparticles: versatile biomaterials for diagnostic and therapeutic applications. Biomater Sci. 2017;5(5):872-886.

6. Kvítek O, Siege J, Hnatowicz V, Švorčík V. Noble metal nanostructures influence of structure and environment on their optical properties. J Nanomater. 2013;2013:1-15.

7. Derkachova A, Kolwas K, Demchenko I. Dielectric function for gold in plasmonics applications: size dependence of plasmon resonance frequencies and damping rates for nanospheres. Plasmonics. 2016;11(3): 941-951.

8. Kelly KL, Coronado E, Zhao LL, Schatz GC. The optical properties of metal nanoparticles: the influence of size, shape, and dielectric environment. J Phys Chem B. 2003;107(3):668-677.

9. Link S, El-Sayed MA. Size and temperature dependence of the plasmon absorption of colloidal gold nanoparticles. J Phys Chem B. 1999; 103(21):4212-4217.

10. Hu M, Chen J, Li Z-Y, et al. Gold nanostructures: engineering their plasmonic properties for biomedical applications. Chem Soc Rev. 2006; 35(11):1084-1094.

11. Yang Y, Wang H. Perspectives of nanotechnology in minimally invasive therapy of breast cancer. $J$ Healthc Eng. 2013;4(1):67-86.

12. Seo SH, Kim BM, Joe A, et al. NIR-light-induced surface-enhanced Raman scattering for detection and photothermal/photodynamic therapy of cancer cells using methylene blue-embedded gold nanorod@SiO2 nanocomposites. Biomaterials. 2014;35(10):3309-3318.

13. Huang P, Lin J, Wang S, et al. Photosensitizer-conjugated silica-coated gold nanoclusters for fluorescence imaging-guided photodynamic therapy. Biomaterials. 2013;34(19):4643-4654.

14. Hu Y, Kanka J, Liu K, Yang Y, Wang H, Du H. Gold nanoring-enhanced generation of singlet oxygen: an intricate correlation with surface plasmon resonance and polyelectrolyte bilayers. $R S C A d v$. 2016;6(106): $104819-104826$.

15. Yang Y, Gao N, Hu Y, et al. Gold nanoparticle-enhanced photodynamic therapy: effects of surface charge and mitochondrial targeting. Ther Deliv. 2015;6(3):307-321. 
16. Oo MKK, Yang Y, Hu Y, Gomez M, Du H, Wang H. Gold nanoparticleenhanced and size-dependent generation of reactive oxygen species from protoporphyrin IX. ACS Nano. 2012;6(3):1939-1947.

17. Yang Y, Hu Y, Du H, Wang H. Intracellular gold nanoparticle aggregation and their potential applications in photodynamic therapy. Chem Commun. 2014;50(55):7287-7290.

18. Hu Y, Yang Y, Wang H, Du H. Synergistic integration of layer-by-layer assembly of photosensitizer and gold nanorings for enhanced photodynamic therapy in the near infrared. ACS Nano. 2015;9(9):8744-8754.

19. Wang C, Cheng L, Liu Y, et al. Imaging-guided $\mathrm{pH}$-sensitive photodynamic therapy using charge reversible upconversion nanoparticles under near-infrared light. Adv Funct Mater. 2013;23(24):3077-3086.

20. Zhang L, Zeng L, Pan Y, et al. Inorganic photosensitizer coupled Gdbased upconversion luminescent nanocomposites for in vivo magnetic resonance imaging and near-infrared-responsive photodynamic therapy in cancers. Biomaterials. 2015;44:82-90.

21. Frangioni JV. In vivo near-infrared fluorescence imaging. Curr Opin Chem Biol. 2003;7(5):626-634.

22. Oo MKK, Yang X, Du H, Wang H. 5-aminolevulinic acid-conjugated gold nanoparticles for photodynamic therapy of cancer. Nanomedicine (Lond). 2008;3(6):777-786.

23. Hu Y, Chou T, Wang H, Du H. Monodisperse colloidal gold nanorings: synthesis and utility for surface-enhanced Raman scattering. J Phys Chem C. 2014;118(29):16011-16018.

24. Hu Y, Yang Y, Wang H, Du H. Colloidal gold nanorings for improved photodynamic therapy through field-enhanced generation of reactive oxygen species. In: Parak WJ, Osinski M, Yamamoto K, editors. SPIE BiOS. Colloidal Nanocrystals for Biomedical Applications VIII. International Society for Optics and Photonics; 2013:85950B. Available from: https://www.spiedigitallibrary.org/conference-proceedings-ofspie/8595/1/Colloidal-gold-nanorings-for-improved-photodynamictherapy-through-field-enhanced/10.1117/12.2001155.short?SSO=1. Accessed November 7, 2017.

25. Draine BT, Flatau PJ. Discrete-dipole approximation for scattering calculations. J Opt Soc Am A. 1994;11(4):1491-1499.

26. Draine BT, Flatau PJ. User guide for the Discrete Dipole Approximation Code DDSCAT.6.0. Source. 2003;3(1994):1-46. Available from: https://arxiv.org/abs/astro-ph/0309069. Accessed November 7, 2017.

27. Johnson PB, Christy RW. Optical constants of the noble metals. Phys Rev B. 1972;6(12):4370-4379.

28. dos Santos AF, Terra LF, Wailemann RAM, et al. Methylene blue photodynamic therapy induces selective and massive cell death in human breast cancer cells. BMC Cancer. 2017;17(1):194.

29. Theodossiou TA, Olsen CE, Jonsson M, Kubin A, Hothersall JS, Berg K. The diverse roles of glutathione-associated cell resistance against hypericin photodynamic therapy. Redox Biol. 2017;12:191-197.

30. Peng Q, Warloe T, Berg K, et al. 5-Aminolevulinic acid-based photodynamic therapy. Clinical research and future challenges. Cancer. 1997; 79(12):2282-2308.

31. Flors C, Fryer MJ, Waring J, et al. Imaging the production of singlet oxygen in vivo using a new fluorescent sensor, Singlet Oxygen Sensor Green. J Exp Bot. 2006;57(8):1725-1734.
32. Jiang W, Kim BYS, Rutka JT, Chan WCW. Nanoparticle-mediated cellular response is size-dependent. Nature Nanotechnology. 2008;3: 145-150.

33. Maisch T, Baier J, Franz B, et al. The role of singlet oxygen and oxygen concentration in photodynamic inactivation of bacteria. Proc Natl Acad Sci US A. 2007;104(17):7223-7228.

34. Rollakanti KR, Kanick SC, Davis SC, Pogue BW, Maytin EV. Techniques for fluorescence detection of protoporphyrin IX in skin cancers associated with photodynamic therapy. Photonics Lasers Med. 2013;2(4): 287-303.

35. Yu BP. Cellular defenses against damage from reactive oxygen species. Physiol Rev. 1994;74(1):139-162.

36. Castano AP, Demidova TN, Hamblin MR. Mechanisms in photodynamic therapy: part two - cellular signaling, cell metabolism and modes of cell death. Photodiagnosis Photodyn Ther. 2005;2(1):1-23.

37. Agostinis $\mathrm{P}, \mathrm{Berg} \mathrm{K}$, Cengel KA, et al. Photodynamic therapy of cancer: an update. CA Cancer J Clin. 2011;61(4):250-281.

38. Oleinick NL, Morris RL, Belichenko I. The role of apoptosis in response to photodynamic therapy: what, where, why, and how. Photochem Photobiol Sci. 2002;1(1):1-21.

39. Ke X, Wang D, Chen C, et al. Co-enhancement of fluorescence and singlet oxygen generation by silica-coated gold nanorods core-shell nanoparticle. Nanoscale Res Lett. 2014;9(1):2492.

40. Liu B, Li C, Cheng Z, Hou Z, Huang S, Lin J. Functional nanomaterials for near-infrared-triggered cancer therapy. Biomater Sci. 2016;4(6): $890-909$.

41. Huang X, El-Sayed IH, Qian W, El-Sayed MA. Cancer cell imaging and photothermal therapy in the near-infrared region by using gold nanorods. J Am Chem Soc. 2006;128(6):2115-2120.

42. Huang X, Jain PK, El-Sayed IH, El-Sayed MA. Plasmonic photothermal therapy (PPTT) using gold nanoparticles. Lasers Med Sci. 2008; 23(3):217-228

43. Pasparakis G. Light-induced generation of singlet oxygen by naked gold nanoparticles and its implications to cancer cell phototherapy. Small. 2013;9(24):4130-4134.

44. Zhao T, Shen X, Li L, et al. Gold nanorods as dual photo-sensitizing and imaging agents for two-photon photodynamic therapy. Nanoscale. 2012;4(24):7712-7719.

45. Vankayala R, Kuo C-L, Sagadevan A, Chen P-H, Chiang C-S, Hwang KC. Morphology dependent photosensitization and formation of singlet oxygen $\left({ }^{1} \Delta_{\mathrm{g}}\right)$ by gold and silver nanoparticles and its application in cancer treatment. J Mater Chem B. 2013;1(35):4379-4387.

46. Vankayala R, Sagadevan A, Vijayaraghavan P, Kuo CL, Hwang KC. Metal nanoparticles sensitize the formation of singlet oxygen. Angew Chem Int Ed Engl. 2011;50(45):10640-10644. 


\section{Supplementary materials}

A
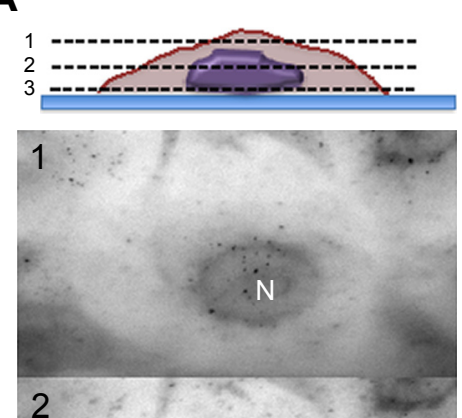

2

$\mathrm{N}$

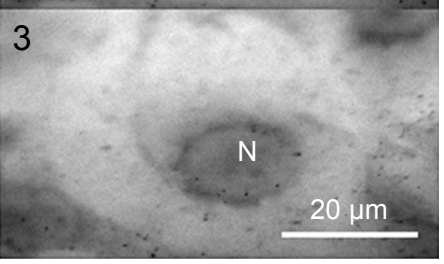

C
B

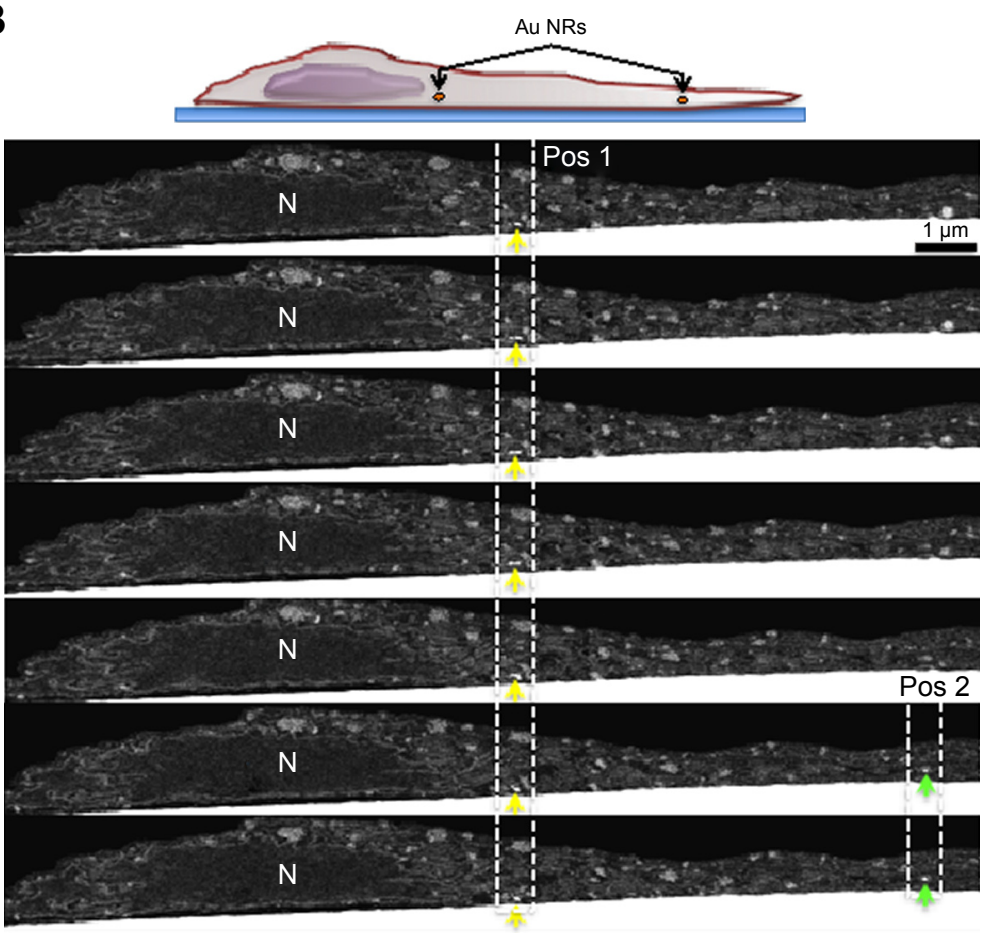

Position 1

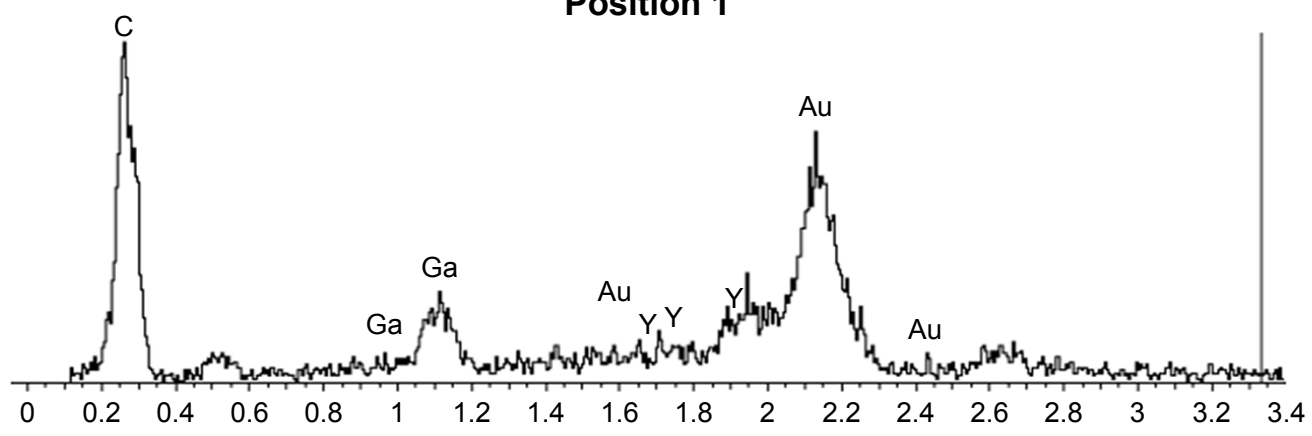

Full scale 159 cts cursor: 3.335 (3 cts)

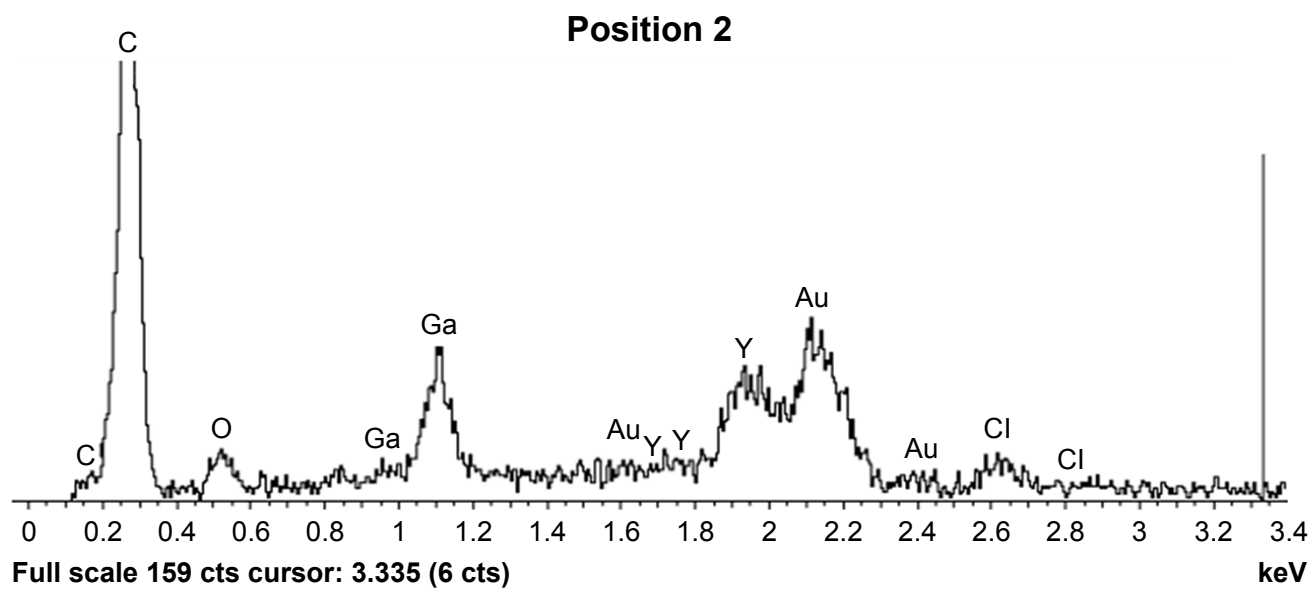

Figure SI (A) Scheme and optical confocal images of MDA-MB-23I cells incubated with Au NRs at three different focus planes. (B) Scheme and focused ion beam scanning electron microscope images of MDA-MB-23I cells incubated with Au NRs. Positions I and 2 show the Au NRs. The interspace between each slice is 30 nm. (C) EDS spectra of position I and position 2.

Abbreviations: Au NR, gold nanoring; cts, counts; EDS, energy dispersive spectroscopy; N, nucleus. 


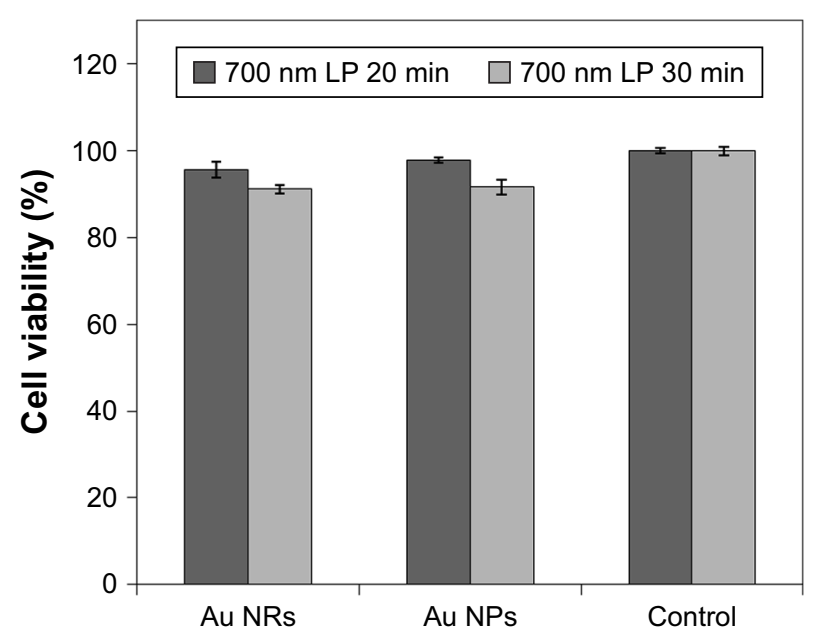

Figure S2 Cell viability of MDA-MB-23I cells after photothermal treatment under near-infrared light $(\lambda>700 \mathrm{~nm})$ irradiation for 20 and $30 \mathrm{~min}$. Cell viability was determined by MTT assay, in which cells were incubated with different gold nanostructures at the same concentration. The culture without gold nanostructures was used as controls.

Abbreviations: Au NP, gold nanoparticle; Au NR, gold nanoring.

\section{Publish your work in this journal}

The International Journal of Nanomedicine is an international, peerreviewed journal focusing on the application of nanotechnology in diagnostics, therapeutics, and drug delivery systems throughout the biomedical field. This journal is indexed on PubMed Central, MedLine, CAS, SciSearch ${ }^{\circledR}$, Current Contents ${ }^{\circledR} /$ Clinical Medicine,
Journal Citation Reports/Science Edition, EMBase, Scopus and the Elsevier Bibliographic databases. The manuscript management system is completely online and includes a very quick and fair peer-review system, which is all easy to use. Visit http://www.dovepress.com/ testimonials.php to read real quotes from published authors. 